\title{
Ocimum gratissimum Aqueous Extract Induces Apoptotic Signalling in Lung Adenocarcinoma Cell A549
}

\author{
Han-Min Chen, ${ }^{1}$ Mu-Jang Lee, ${ }^{2}$ Cheng-Yi Kuo, ${ }^{3}$ Pei-Lin Tsai, ${ }^{4}$ Jer-Yuh Liu, ${ }^{5}$ \\ and Shao-Hsuan Kao ${ }^{4,6,7}$ \\ ${ }^{1}$ Department of Life Science, Fu-Jen Catholic University, Taipei 24205, Taiwan \\ ${ }^{2}$ Department of Internal Medicine, Division of Cardiology, Tian-Sheng Memorial Hospital, Pingtung 92843, Taiwan \\ ${ }^{3}$ Multi Chemical Laboratory, SGS Taiwan Ltd., Taipei 24803, Taiwan \\ ${ }^{4}$ Institute of Biochemistry and Biotechnology, Chung Shan Medical University, Taichung City 40201, Taiwan \\ ${ }^{5}$ Graduate Institute of Cancer Biology, China Medical University and Hospital, Taichung City 40402, Taiwan \\ ${ }^{6}$ Clinical Laboratory, Chung Shan Medical University Hospital, Taichung City 40201, Taiwan \\ ${ }^{7}$ Institutes of Biochemistry and Biotechnology, Chung Shan Medical University, No. 110, Sec. 1, Jianguo N. Road, \\ Taichung 40201, Taiwan
}

Correspondence should be addressed to Shao-Hsuan Kao, kaosh@csmu.edu.tw

Received 29 April 2010; Accepted 31 August 2010

Copyright (c) 2011 Han-Min Chen et al. This is an open access article distributed under the Creative Commons Attribution License, which permits unrestricted use, distribution, and reproduction in any medium, provided the original work is properly cited.

Ocimum gratissimum (OG) is widely used as a traditional herb for its antibacterial activity in Taiwan. Recently, antitumor effect of OG on breast cancer cell is also reported; however, the effects of OG on human pulmonary adenocarcinoma cell A549 remain unclear. Therefore, we aimed to investigate whether aqueous OG extract (OGE) affects viability of A549 cells and the signals induced by OGE in A549 cells. Cell viability assays revealed that OGE significantly and dose-dependently decreased the viability of A549 cell but not that of BEAS-2B cell. Morphological examination and DAPI staining indicated that OGE induced cell shrinkage and DNA condensation for A549 cells. Further investigation showed that OGE enhanced activation of caspase-3, caspase-9 and caspase- 8 and increased protein level of Apaf- 1 and Bak, but diminished the level of Bcl-2. Additionally, OGE inhibited the phosphorylation of extracellular signal-regulated kinase (ERK) yet enhanced the phosphorylation of c-Jun N-terminal kinase (JNK) and p38 MAP kinase (p38). In conclusion, our findings indicate that OGE suppressed the cell viability of A549 cells, which may result from the activation of apoptotic signaling and the inhibition of anti-apoptotic signaling, suggesting that OGE might be beneficial to lung carcinoma treatment.

\section{Introduction}

Lung adenocarcinoma is the major cause of cancer-related mortality worldwide $[1,2]$. Despite clinical success of concurrent therapeutic approaches against lung cancer including chemotherapy and radiotherapy, marked chances of undesirable and adverse side effects caused by such therapies need to be managed. Use of medicinal plants in chemoprevention is considered as an ideal treatment with good efficacy and few side effects compared with allopathic medicine [3]. Mounting evidences have shown that dietary intake of phytochemicals, an important group of chemopreventive agents, reduces the risk of cancer [4] and has antitumor potential against lung cancer [5].
Chemopreventive agents are compounds that prevent development of cancer. Their preventive effects are attributed to [1] intervening in interaction of the carcinogen with cellular DNA, [2] altering intracellular signaling pathways as results of stopping progression of an initiated cell through preneoplastic changes into a malignant cell, [3] inhibiting angiogenesis, [4] inducing cell cycle arrest, and [5] triggering apoptosis. It is believed that the apoptosis induced by chemopreventive agents may not only inhibit the carcinogenesis induced by carcinogens, but also may suppress the growth of tumor and enhance the cytotoxic effects of antitumor drug on tumor, which plays a pivotal role in the antitumor therapies [6]. 
The genus Ocimum, belonging to the family Labiatae, is widely found in tropical and subtropical regions. The widespread plant is known for its chemopreventive, anticarcinogenic, free radical scavenging, and other pharmacological properties and used as a traditional herb in European and Asian countries since ancient times [7]. It has been prepared in a variety of forms for consumption. The aqueous leaf extract and seed oil are reported to show chemopreventive and antiproliferative activity on Hela cells [8]. Ethanolic extract of Ocimum leaf also has been shown to have significant modulatory influence on carcinogen metabolizing enzymes including cytochrome P450, cytochrome b5, and aryl hydrocarbon hydroxylase, glutathione-S-transferase [9, 10]. Additionally, Ocimum sanctum prepared in the form of fresh leaf paste, aqueous, and ethanolic extract has been reported to reduce the incidence of papillomas and squamous cell carcinoma in carcinogen-treated hamsters with an observation that the aqueous extract exerts more profound effect than the other two forms [11]. Nevertheless, the mechanisms of aqueous extract of Ocimum gratissimum (OGE) underlying its anticancer property remain sketchy.

In the present study, the anticancer effects of OGE were investigated using human lung carcinoma A549 cells. The effects of OGE on cell viability and apoptosis of A549 cell were determined by measuring the activity of mitochondrial malate dehydrogenase and the DNA condensation, respectively. The OGE-induced caspase activation cascades and kinase signaling, including caspase-3, caspase8, caspase-9, apaf- $1, \mathrm{Bcl}-2$, Bak, extracellular signal-regulated kinase (ERK), Akt, c-Jun N-terminal kinase (JNK), and p38 mitogen-activated protein (MAP) kinase (p38), were elucidated using immunological approaches

\section{Materials and Methods}

2.1. Materials. [3-(4,5-dimethylthiazol-2-yl)-2,5-diphenyltetrazolium bromide (MTT), 4'-6-Diamidino-2-phenylindole (DAPI), penicillin, and streptomycin were purchased from Sigma (St. Louis, MO).

Dulbecco's modified Eagle's medium (DMEM), fetal bovine serum (FBS), and trypsin-EDTA were purchase from Gibco BRL (Gaithersburg, MD). Antibodies against caspase3 , caspase-8, caspase-9, apaf-1, Bak, Bcl-2, phosphorylatedERK1/2 (p-ERK1/2), ERK1/2, phosphorylated-JNK (p-JNK), JNK, phosphorylated-p38 (p-p38), and p38 were purchased from Cell Signaling Technologies (Beverly, MA). Antibodies against $\beta$ actin and Glyceraldehyde 3-phosphate dehydrogenase (GAPDH) were obtained from Sigma. HRP-conjugated secondary antibodies against mouse IgG and rabbit IgG were purchased from Abcam Inc. (Cambridge, UK). The lung adenocarcinoma cell A549 was obtained from American Type Culture Collection (ATCC; Rockville, MD).

2.2. Preparation of OGE and Composition Analysis. Leaves of Ocimum gratissimum Linn were harvested and washed with distilled water followed by homogenization with distilled water using polytron. The homogenate was incubated at $95^{\circ} \mathrm{C}$ for 1 hour (h) and then filtered through two layers of gauze. The filtrate was centrifuged at $20,000 \mathrm{~g}, 4^{\circ} \mathrm{C}$ for
15 minute to remove insoluble pellets and the supernatant (OGE) was thereafter collected, lyophilized, and stored at $-70^{\circ} \mathrm{C}$ until use.

The content of polyphenol in OGE was analyzed as previously described in [12] and revealed the final extract (OGE) contained $11.1 \%$ polyphenolic acid and $4.5 \%$ flavonoids.

2.3. Cell Culture and Experimental Treatments. Lung adenocarcinoma cell A549 and SV40-transformed normal epithelial cell BEAS-2B were maintained in DMEM supplemented with $10 \% \mathrm{FBS}$ and $100 \mu \mathrm{g} / \mathrm{ml}$ penicillin/streptomycin at $37^{\circ} \mathrm{C}$ in a humidified atmosphere containing $5 \% \mathrm{CO}_{2}$. Cells were seeded in 6-well culture plates at an initial density of $1 \times$ $10^{5}$ cells $/ \mathrm{ml}$ and grown to approximately $80 \%$ confluence. OGE treatments were performed by incubating cells with serial concentrations of OGE ( $w / v)$ in serum-free DMEM for $48 \mathrm{~h}$. After the OGE treatments, the cells were washed with phosphate-buffered saline (PBS; $25 \mathrm{mM}$ sodium phosphate, $150 \mathrm{mM} \mathrm{NaCl}, \mathrm{pH}$ 7.2) and collected for following analyses.

2.4. DAPI Staining. DAPI staining was performed to assess morphological changes in the chromatin structure of A549 cells undergoing apoptosis as previously described in [13]. Briefly, cells were trypsinized, mounted on glass slides, and fixed in $4 \%$ paraformaldehyde for 30 minutes. followed by staining with $1 \mu \mathrm{g} / \mathrm{ml}$ DAPI for 30 minutes Apoptosis was characterized by chromatin condensation and fragmentation when examined by fluorescence microscopy. The incidence of apoptosis in each treatment was analyzed by counting 300 cells and presented in the percentage of apoptotic cells.

2.5. Cell Viability Assay. Cell viability was determined by MTT assay [14] in the absence or presence of 50 or $100 \mu \mathrm{g} / \mathrm{ml}$ OGE. After $48 \mathrm{~h}$ treatments, culture medium was aspirated and cells were incubated with MTT $(0.5 \mathrm{mg} / \mathrm{ml})$ at $37^{\circ} \mathrm{C}$ for $4 \mathrm{~h}$. The viable cell number was directly proportional to the production of formazan, which was dissolved in isopropanol and determined by measuring the absorbance at $570 \mathrm{~nm}$ using a microplate reader (SpectraMAX $360 \mathrm{pc}$, Molecular Devices, Sunnyvale, CA).

2.6. Immunoblotting. A549 cells were washed with PBS and lysed with lysis buffer $(50 \mathrm{mM}$ Tris- $\mathrm{HCl}, \mathrm{pH} 7.5,150 \mathrm{mM}$ $\mathrm{NaCl}, 1 \%$ Nonidet P-40, $1 \mathrm{mM}$ phenylmethylsulfonyl fluoride, $1 \mathrm{mM}$ sodium fluoride, and $10 \mu \mathrm{g} / \mathrm{ml}$ aprotinin and leupeptin). The lysates were incubated on ice for 30 minutes and centrifuged at 20,000 $\mathrm{g}$ for 15 minutes. The supernatants were collected and measured for protein concentration using Bradford method. Crude proteins ( $30 \mu \mathrm{g}$ per lane) were subjected to a $12.5 \%$ SDS-polyacrylamide gel, and then transferred onto a nitrocellulose membrane (Millipore, Bedford, MA). The blotted membrane was blocked with $5 \% \mathrm{w} / \mathrm{v}$ skimmed milk in PBS, and then incubated for $2 \mathrm{~h}$ with $1 / 1000$ dilution of antibodies against human caspase-3, caspase-8, caspase-9, apaf-1, Bak, Bcl-2, p-ERK1/2, ERK1/2, p-JNK, JNK, p-p38, p38, $\beta$-actin, and GAPDH, respectively. Antigen-antibody complex were detected using $1 / 2000$ dilution of peroxidase-conjugated secondary antibodies and displayed using ECL chemiluminescence reagent (Millipore). 


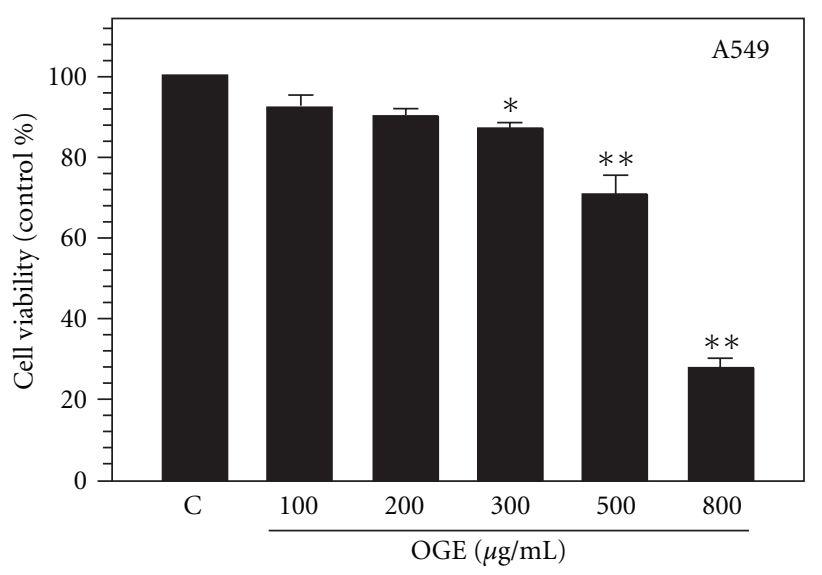

(a)

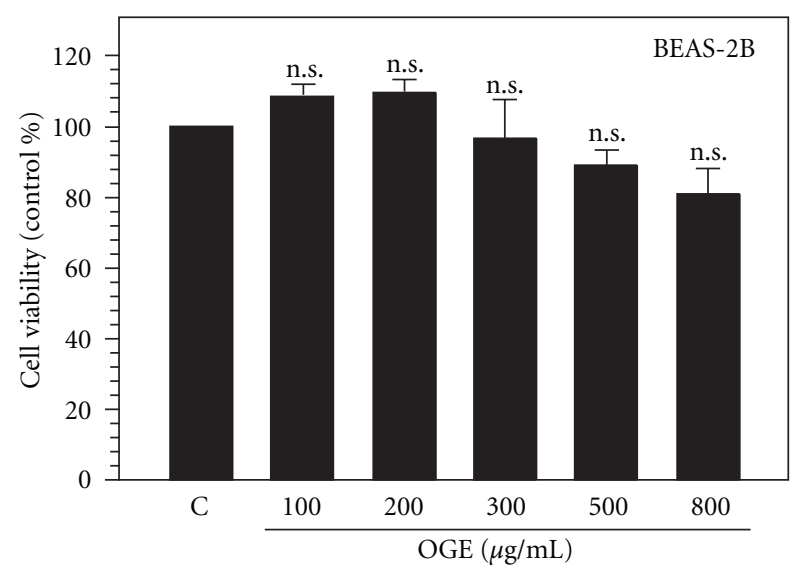

(b)

FIGURE 1: OGE diminished cell viability of A549 cell. The cell viability of A549 cells (a) and BEAS-2B cells (b) treated with serial concentrations of OGE $(10,50,100,200$ and $300 \mu \mathrm{g} / \mathrm{ml})$ for $48 \mathrm{~h}$ was determined. Data were expressed as mean \pm SEM for 3 independent experiments. ${ }^{*} P<.05$ and, ${ }^{* *} P<.01$ as comparing to control (c). n.s., no statistical significance.

2.7. Statistical Analysis. Data were expressed as means \pm SEMs of the three independent experiments. Statistical significance analysis was determined by using 1-way ANOVA followed by Dunnett for multiple comparisons with the control. The differences were considered significant for $P$ values less than 05 .

\section{Results}

3.1. Growth Suppression of Human Lung Carcinoma A549 Cells by OGE Administration. To examine antitumor activity of OGE, both lung carcinoma cell A549 and epithelial cell BEAS-2B were treated with serial concentrations of OGE $(100,200,300,500$, and $800 \mu \mathrm{g} / \mathrm{ml})$ for $48 \mathrm{~h}$, and subsequently analyzed for the cell viability. As shown in Figure 1(a), the viability of A549 cells exposed to OGE for 48 hours was found decreased in association with the concentration of OGE in a dose-dependent fashion. The viability was significantly decreased to $92.3 \pm 3.2 \%, 90.1 \pm$ $1.4 \%, 87.2 \pm 1.2 \%, 70.6 \pm 3.3 \%$, and $27.5 \pm 1.4 \%$ of control with $100,200,300,500$ and $800 \mu \mathrm{g} / \mathrm{ml}$ OGE, respectively $(P<.05$ as compared to control). Interestingly, the cell viability of BEAS-2B was insignificantly affected by OGE treatment. However, the variation of the viability was not statistically significant $(.061<P<.121$ as compared to control) (Figure 1(b)). Accordingly, the findings showed that 500 , and $800 \mu \mathrm{g} / \mathrm{ml}$ OGE treatment significantly suppressed the viability of A549 cells but not that of BEAS-2B.

\subsection{OGE-Induced Morphological Alteration and DNA Con-} densation in A549 Cells. To explore the mechanism underlying cell death in presence of OGE, cell morphology and chromatin condensation of OGE-treated A549 cells was examined. As shown in Figure 2(a), OGE treatment altered the morphology of A549 cells as results of cell shrinkage and detachment (left panel). Additionally, a phenomenon of chromatin condensation was observed in the A549 cells treated with OGE in a dose-dependent manner (right panel).
The incidence of DNA condensation was quantified, showing that 500 and $800 \mu \mathrm{g} / \mathrm{ml}$ OGE treatment resulted in significant chromatin condensation $(17.5 \pm 1.7 \%$ and $56.8 \pm 2.3 \%, P<$ .01 as compared to control) in A549 cell (Figure 2(b)).

\subsection{Activation of Both Intrinsic and Extrinsic Apoptotic Path-} way in A549 Cells following OGE Treatment. To discriminate the apoptotic mechanisms induced by OGE treatment, the activations of intrinsic and extrinsic caspase cascades were investigated. As shown in Figure 3, the level of procaspase-3, an important effecter caspase, was decreased in response to OGE treatment in a dose-dependent manner. Concomitant with the decrease of procaspase-3, the increase in cleaved caspase- 3 in A549 cells was observed in association with OGE treatment in a dose-dependent fashion. The content of caspase-9 and Apaf-1, two upstream activators of procaspase- 3 in intrinsic pathway, were further investigated. The treatment of A549 cells with OGE resulted in the decrease of procaspase- 9 and the increase of its cleavage product. The level of Apaf-1 was also dose-dependently increased by OGE treatment (Figure 3).

The effect of OGE treatment on caspase-8, an upstream activator of caspase- 3 in extrinsic pathway, was also investigated. Although no obvious change in the level of procaspase- 8 was observed in presence of OGE, the levels of two cleaved caspase- 8 ( $43 \mathrm{kDa}$ and $12 \mathrm{kDa}$ ) were remarkably increased by OGE treatment (Figure 4).

3.4. Modulation of Bak and Bcl-2 in OGE-Treated A549 Cells. To further elucidate the putative mechanism underlying the OGE-associated apoptotic signaling, the levels of proapoptotic proteins Bak and antiapoptotic proteins Bcl2 at various concentrations of OGE were examined. After being normalized and verified with GAPDH, expression of Bax was increased remarkably in a dose-dependent manner. Moreover, an obvious decrease in the Bcl-2 protein level was detected in the OGE-treated A549 cells as compared to the control (Figure 5(a)). 


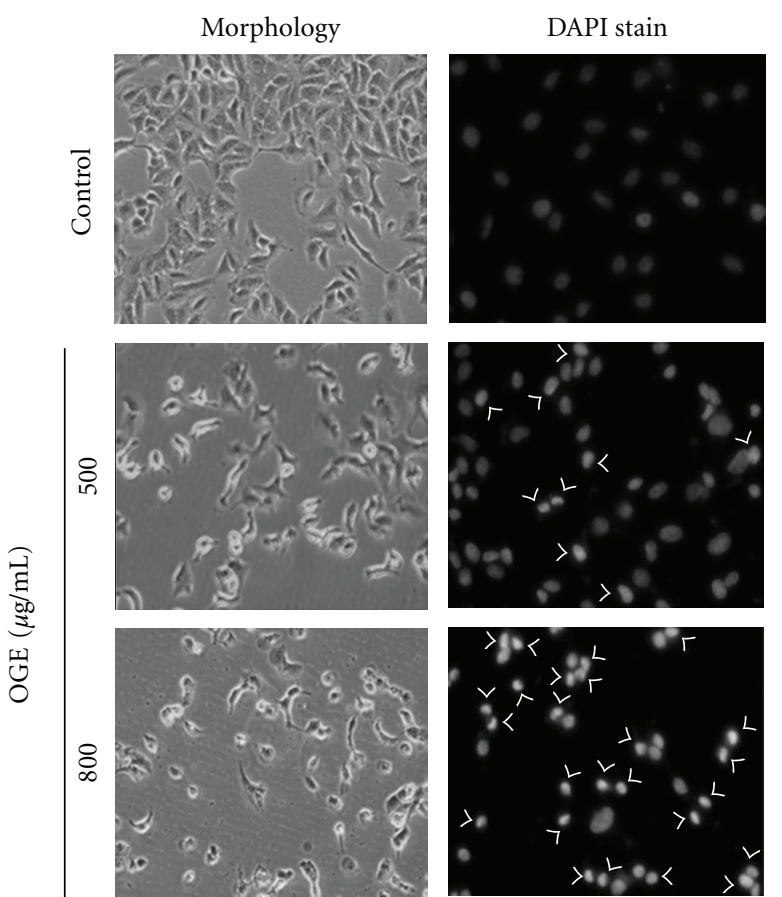

(a)

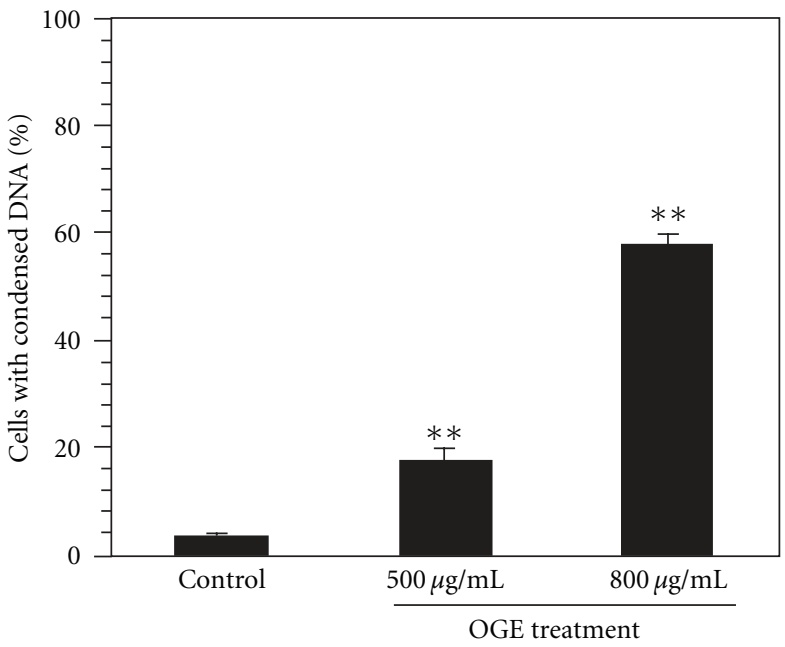

(b)

FIGURE 2: OGE altered cell morphology and induced DNA condensation of A549 cell. A549 cells were treated with 0,500 and $800 \mu \mathrm{g} / \mathrm{ml} \mathrm{OGE}$ for $48 \mathrm{~h}$ and then stained with DAPI. (a) The cell morphology and the DNA condensation was photographed by fluorescence microscopy (200x). The cells presented DNA condensation were indicated by arrow. (b) The incidence of DNA condensation was determined for the A549 cells with different treatments. Data were expressed as mean \pm SEM for 3 independent experiments.

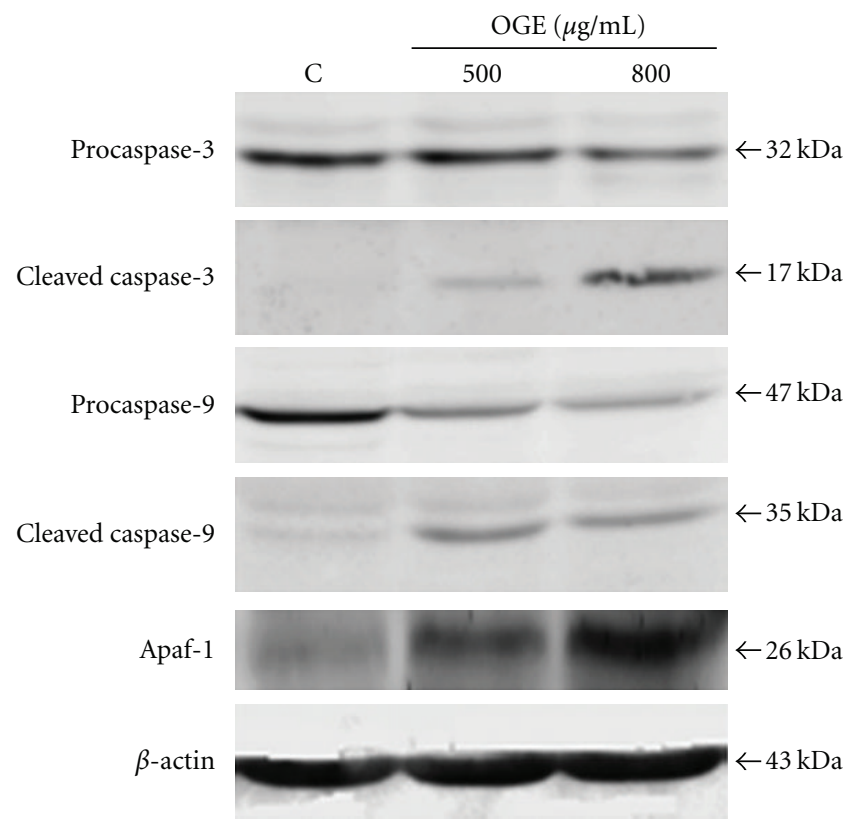

FIGURE 3: OGE induced activation of intrinsic/mitochondrial apoptotic pathway. A549 cells were treated with 0,500 and $800 \mu \mathrm{g} / \mathrm{ml}$ for $48 \mathrm{hr}$, and then were lyzed for the determination of protein levels of caspase- 3 , cleaved caspase-3, caspase- 9 , cleaved caspase- 9 and Apaf- 1 by immunoblotting. $\beta$-actin was used as control. The apparent molecular weights for detected proteins were indicated.

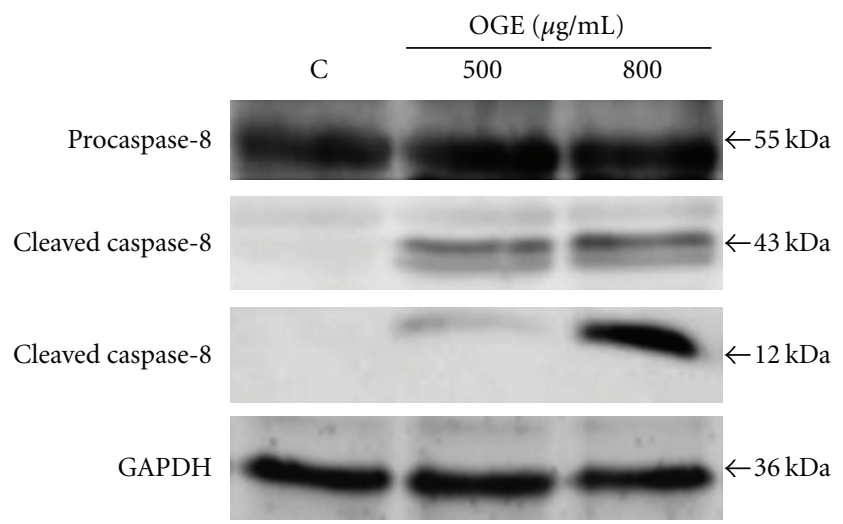

FIGURE 4: OGE induced activation of caspase- 8 . A549 cells were treated with 0,500 and $800 \mu \mathrm{g} / \mathrm{ml}$ for $48 \mathrm{hr}$, and then were lyzed for the determination of protein levels of caspase- 8 and cleaved caspase8 by immunoblotting. $\beta$ actin was used as control. The apparent molecular weights for detected proteins were indicated.

Quantitative Bak and Bcl-2 expression after being standardized to GAPDH $(n=3)$ was shown in Figure 5(b). Expression of Bak was increased significantly upon OGE treatment, whereas the significant decrease in expression of Bcl-2 in A549 cells. With $800 \mu \mathrm{g} / \mathrm{ml}$ OGE treatment, the level of Bak and Bcl-2 was increased to $321.3 \pm 11.4 \%$ and decreased to $50.2 \pm 2.2 \%$, respectively $(P<.01$ as compared to control). 


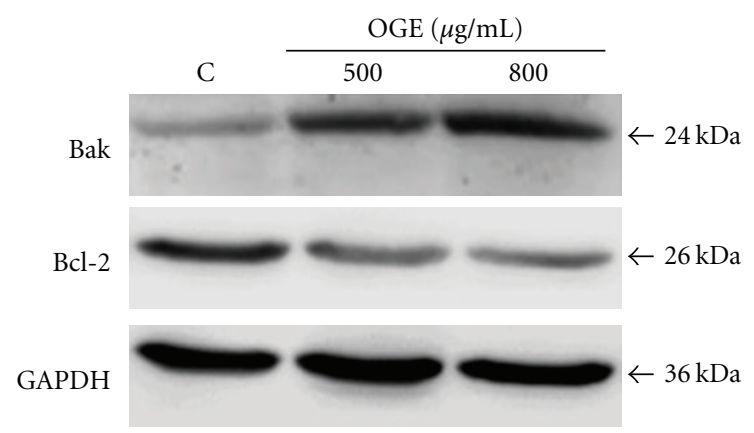

(a)

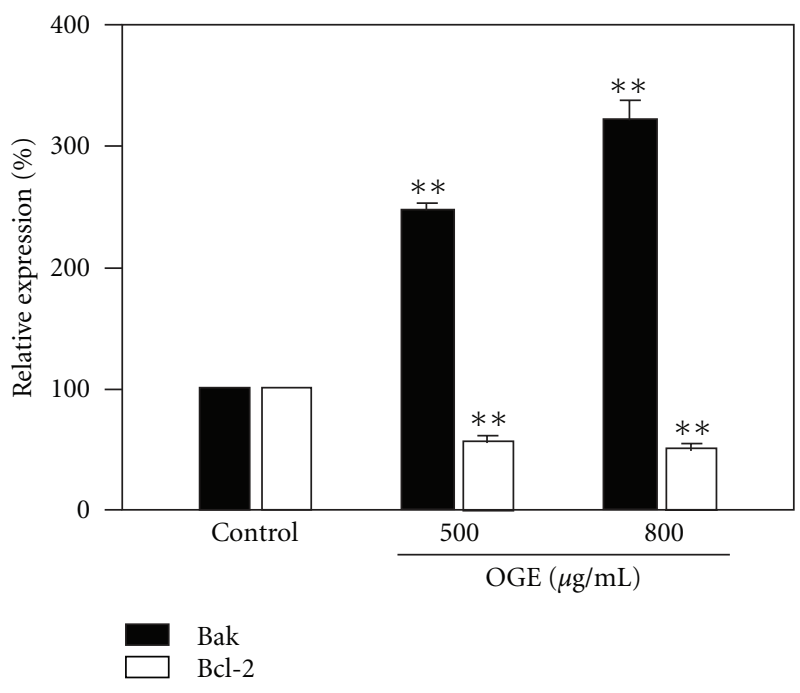

(b)

FIGURE 5: OGE enhanced protein level of Bak and diminished protein level of Bcl-2. A549 cells were treated with 0, 500, and $800 \mu \mathrm{g} / \mathrm{ml}$ for $48 \mathrm{hr}$, and then were lyzed for the determination of protein levels by immunoblotting. (a) The expression levels of Bak and Bcl-2 were determined. GAPDH was used as control. (b) The expression levels of Bak and Bcl-2 were quantitatively expressed after being standardized to GAPDH. Data are expressed as mean \pm SEM for 3 independent experiments for each concentration point. ${ }^{* *} P<.01$ as compared with control.

\subsection{Activation of JNK and p38 but Not ERK MAP Kinase} in A549 Cells following OGE Treatment. MAP kinases have been widely reported for their involvements in the survival, proliferation, differentiation, and apoptosis in different cancer cells [15]. Therefore, the influence of OGE treatment on activation of three important MAP kinases, ERK, JNK, and p38, were further investigated. As shown in Figure 6, remarkable phosphorylation of ERK, but not JNK and p38, was detected in the control A549 cells. OGE treatment significantly inhibited the phosphorylation of ERK and enhanced the phosphorylation of JNK and p38.

\section{Discussion}

Aberrant cells such as mutated or proliferating neoplastic cells are removed by programmed cell death, namely,

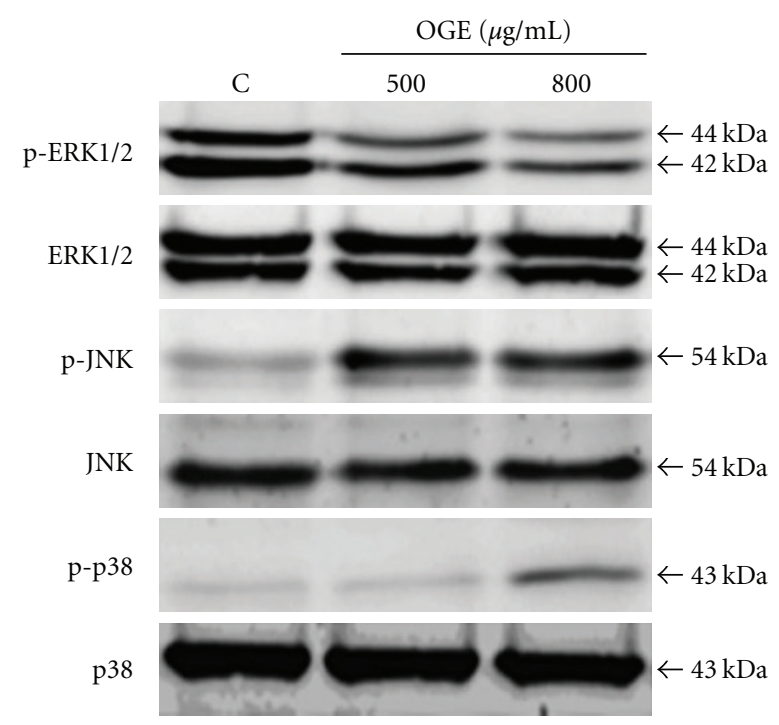

FIGURE 6: OGE inhibited phosphorylation of Erk but enhanced phosphorylation of JNK and p38. A549 cells were treated with 0,500 and $800 \mu \mathrm{g} / \mathrm{ml}$ for $48 \mathrm{hr}$, and then were lyzed for the determination of protein levels by immunoblotting. The levels of p-ERK1/2, ERK1/2, p-JNK, JNK, p-p38, and p38 were presented.

apoptosis [16]. Two well-known pathways, extrinsic and intrinsic pathways, are responsible for triggering apoptosis [17]. In the case of the intrinsic pathway, a release of cytochrome $\mathrm{C}$ from mitochondria results in binding to Apaf- 1 and subsequently leads to activation of procaspase- 9 and following caspase-3 [18]. Activated caspase-3 exerts as the key executioner of apoptosis to induce the cleavage and inactivation of key cellular protein $[18,19]$. In the present study, it is demonstrated that OGE treatment increased the Apaf- 1 expression level and activated the caspase- 9 and 3 cascade. Additionally, it is known that caspase-3 can be activated by caspase- 8 through the extrinsic pathway [20]. Our results showed that OGE treatment simultaneously induced the activation of caspase- 8 . These findings indicate that both activations of the intrinsic and extrinsic pathway are of responses to exposure to OGE in A549 cells as results of apoptosis.

MAP kinase cascades consist of a core of three protein kinases such as ERK1/2, p38, and JNK pathways [21, 22]. Thus, to understand the molecular mechanism of OGE, the potential involvement of MAP kinase pathway in OGEinduced apoptosis was investigated by immunoblotting. The aberrant expressions of Akt and ERK are known as a prominent feature of many human cancers including nonsmall cell lung cancer [23]. Our findings are consistent with the aberration that a relative high level of ERK phosphorylation in the control A549 cells, which may contribute to the malignancy and high frequency of metastasis of lung cancer. Interestingly, our results that ERK phosphorylation in A549 cells was significantly inhibited in presence of OGE suggest that OGE treatment could have suppressive influence on the constitutive survival signaling for A549 cells. Moreover, the JNK and p38 phosphorylation in A549 cells was found 


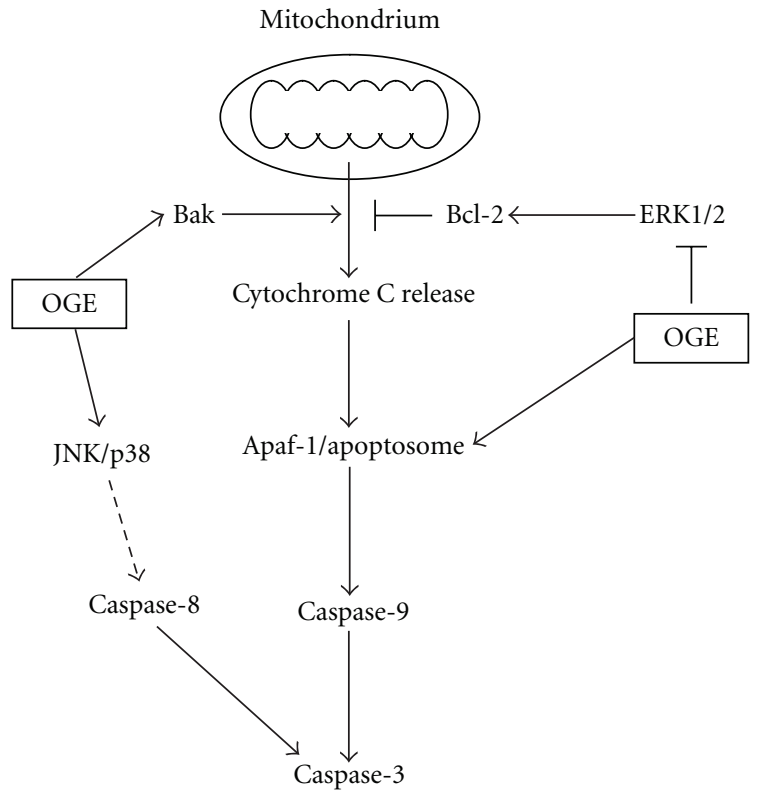

Figure 7: Proposed model for the antitumoral activity of Ocimum gratissimum extracts on the human lung adenocarcinoma A549. Our data demonstrated that Ocimum gratissimum extract induces activation of caspase- 3 , caspase- 9 , and caspase- 8 , which may attribute to the increase of Apaf-1 and Bak, the decrease of antiapoptotic Bcl-2, as well as the inhibition of ERK1/2 survival signaling and the enhancement of JNK and p38 stress signal cascades.

enhanced in response to OGE treatment, which plays an important role in apoptotic signaling through regulating the activities of pre-existing $\mathrm{Bcl}-2$ family proteins and mediating caspase activation [12].

Recently, several lines of evidence indicate that extracts of Ocimum species possess antitumor effects. Ethanolic extract of Ocimum sanctum has been reported to induce apoptosis of A549 cell via the intrinsic/mitochondria-dependent pathway [24] and chemical-induced gastric carcinogenesis via inhibition of proliferation, angiogenesis, and apoptosisassociated proteins [25]. Essential oil from Ocimum sanctum also has been shown to exert cytotoxic and apoptotic activity to human colorectal adenocarcinoma cell COLO 205 [26]. Moreover, Ocimum sanctum combining with Azadirachta indica is reported to have synergistic chemopreventive effects on chemical-induced gastric carcinogenesis, which may be attributed to their anti-oxidant, antiangiogenic, antiproliferative, and apoptosis inducing properties [27]. These findings indicate that Ocimum spp. extracts possess potent antitumoral activity through inducing apoptosis, inhibiting proliferation and suppressing angiogenesis.

In the present study, we show that OGE activates both intrinsic and extrinsic pathway in A549 cells. Aqueous extract of OG leaf has been reported to inhibit the growth and the migration of breast cancer cell MDA-MB-231 [28]. With the treatment of $0.5 \%(5 \mathrm{mg} / \mathrm{ml})$ aqueous extract of $\mathrm{OG}$ leaf for $72 \mathrm{~h}$, the cell viability of MDA-MB-231 was reduced to approximately $60 \%$ of the control. Our results reveal that the lower concentration of OGE (500 and $800 \mu \mathrm{g} / \mathrm{ml}$ ) significantly diminishes the cell viability of A549 to 47\% and $36 \%$ of the control. These findings suggest that lung adenocarcinoma cells are more susceptible to OGE treatment than breast cancer cells.

In conclusion, the present study provides evidences that OGE treatments significantly alter viability of lung adenocarcinoma A549 cells through a synergy of induction of apoptotic signaling and suppression of antiapoptotic signaling, as shown in Figure 7. Moreover, OGE treatment simultaneously inhibits the activation of ERK and enhances the activation of JNK and p38, which is consistent with the enhanced apoptotic signaling and reduced antiapoptotic signaling based on their well-known effects on these signal cascades (Figure 7). By manipulating both arms of apoptotic and antiapoptotic pathway, OGE represents a promisingly effective chemopreventive agent against lung adenocarcinoma.

\section{Acknowledgments}

This paper was partly supported by Grant NSC99-2320-B040-003-MY3 from the National Science Council, Taiwan and by the Taiwan Department of Health Clinical Trial and Research Center of Excellence (DOH99-TD-B-111-004) and in part by the Taiwan Department of Health Cancer Research Center of Excellence (DOH99-TD-C-111-005).

\section{References}

[1] A. Jemal, R. Siegel, E. Ward et al., "Cancer statistics, 2006," Ca: A Cancer Journal for Clinicians, vol. 56, no. 2, pp. 106-130, 2006.

[2] D. M. Parkin, "Global cancer statistics in the year 2000," Lancet Oncology, vol. 2, no. 9, pp. 533-543, 2001.

[3] W. Zhu, X.-M. Wang, L. Zhang, X.-Y. Li, and B.-X. Wang, "Pharmacokinetic of Rhein in healthy male volunteers following oral and retention enema administration of rhubarb extract: a single dose study," American Journal of Chinese Medicine, vol. 33, no. 6, pp. 839-850, 2005.

[4] W. C. Willett, "Diet and health: what should we eat?" Science, vol. 264, no. 5158, pp. 532-537, 1994.

[5] E. T. H. Fontham, "Protective dietary factors and lung cancer," International Journal of Epidemiology, vol. 19, no. 1, pp. S32S42, 1990 .

[6] J. Clark and M. You, "Chemoprevention of lung cancer by tea," Molecular Nutrition and Food Research, vol. 50, no. 2, pp. 144$151,2006$.

[7] S. K. Gupta, J. Prakash, and S. Srivastava, "Validation of traditional claim of Tulsi, Ocimum sanctum Linn. as a medicinal plant," Indian Journal of Experimental Biology, vol. 40, no. 7, pp. 765-773, 2002.

[8] J. Prakash and S. K. Gupta, "Chemopreventive activity of Ocimum sanctum seed oil," Journal of Ethnopharmacology, vol. 72, no. 1-2, pp. 29-34, 2000.

[9] D. R. Gang, T. Beuerle, P. Ullmann, D. Werck-Reichhart, and E. Pichersky, "Differential production of meta hydroxylated phenylpropanoids in sweet basil peltate glandular trichomes and leaves is controlled by the activities of specific acyltransferases and hydroxylases," Plant Physiology, vol. 130, no. 3, pp. 1536-1544, 2002. 
[10] S. Banerjee, R. Prashar, A. Kumar, and A. R. Rao, "Modulatory influence of alcoholic extract of Ocimum leaves on carcinogen-metabolizing enzyme activities and reduced glutathione levels in mouse," Nutrition and Cancer, vol. 25, no. 2, pp. 205-217, 1996.

[11] K. Karthikeyan, P. Ravichandran, and S. Govindasamy, "Chemopreventive effect of Ocimum sanctum on DMBAinduced hamster buccal pouch carcinogenesis," Oral Oncology, vol. 35, no. 1, pp. 112-119, 1999.

[12] C. Tournier, P. Hess, D. D. Yang et al., "Requirement of JNK for stress-induced activation of the cytochrome c-mediated death pathway," Science, vol. 288, no. 5467, pp. 870-874, 2000.

[13] C.-H. Peng, C.-N. Huang, S.-P. Hsu, and C.-J. Wang, "Pentaacetyl geniposide induce apoptosis in C6 glioma cells by modulating the activation of neutral sphingomyelinase-induced p75 nerve growth factor receptor and protein kinase $\mathrm{C} \delta$ pathway," Molecular Pharmacology, vol. 70, no. 3, pp. 997$1004,2006$.

[14] F. Denizot and R. Lang, "Rapid colorimetric assay for cell growth and survival-modifications to the tetrazolium dye procedure giving improved sensitivity and reliability," Journal of Immunological Methods, vol. 89, no. 2, pp. 271-277, 1986.

[15] E. F. Wagner and Á. R. Nebreda, "Signal integration by JNK and p38 MAPK pathways in cancer development," Nature Reviews Cancer, vol. 9, no. 8, pp. 537-549, 2009.

[16] J. A. Hickman, "Apoptosis induced by anticancer drugs," Cancer and Metastasis Reviews, vol. 11, no. 2, pp. 121-139, 1992.

[17] L. E. Broker, F. A. E. Kruyt, and G. Giaccone, "Cell death independent of caspases: a review," Clinical Cancer Research, vol. 11, no. 9, pp. 3155-3162, 2005.

[18] N. A. Thornberry and Y. Lazebnik, "Caspases: Enemies within,” Science, vol. 281, no. 5381, pp. 1312-1316, 1998.

[19] B. B. Wolf and D. R. Green, "Suicidal tendencies: apoptotic cell death by caspase family proteinases," Journal of Biological Chemistry, vol. 274, no. 29, pp. 20049-20052, 1999.

[20] G. M. Cohen, "Caspases: the executioners of apoptosis," Biochemical Journal, vol. 326, no. 1, pp. 1-16, 1997.

[21] G. Pearson, F. Robinson, T. B. Gibson et al., "Mitogenactivated protein (MAP) kinase pathways: regulation and physiological functions," Endocrine Reviews, vol. 22, no. 2, pp. 153-183, 2001.

[22] M. Raman, W. Chen, and M. H. Cobb, "Differential regulation and properties of MAPKs,” Oncogene, vol. 26, no. 22, pp. 3100-3112, 2007.

[23] S. H. Lee, H. S. Kim, W. S. Park et al., "Non-small cell lung cancers frequently express phosphorylated Akt; an immunohistochemical study," APMIS, vol. 110, no. 7-8, pp. 587-592, 2002.

[24] V. Magesh, J.-C. Lee, S. A. Kwang et al., "Ocimum sanctum induces apoptosis in A549 lung cancer cells and suppresses the in vivo growth of lewis lung carcinoma cells," Phytotherapy Research, vol. 23, no. 10, pp. 1385-1391, 2009.

[25] P. Manikandan, P. Vidjaya Letchoumy, D. Prathiba, and S. Nagini, "Proliferation, angiogenesis and apoptosis-associated proteins are molecular targets for chemoprevention of MNNG-induced gastric carcinogenesis by ethanolic Ocimum sanctum leaf extract," Singapore Medical Journal, vol. 48, no. 7, pp. 645-651, 2007.

[26] M. Sharma, S. K. Agrawal, P. R. Sharma, B. S. Chadha, M. K. Khosla, and A. K. Saxena, "Cytotoxic and apoptotic activity of essential oil from Ocimum viride towards COLO 205 cells," Food and Chemical Toxicology, vol. 48, no. 1, pp. 336-344, 2010.
[27] P. Manikandan, P. Vidjaya Letchoumy, D. Prathiba, and S. Nagini, "Combinatorial chemopreventive effect of Azadirachta indica and Ocimum sanctum on oxidant-antioxidant status, cell proliferation, apoptosis and angiogenesis in a rat forestomach carcinogenesis model," Singapore Medical Journal, vol. 49, no. 10, pp. 814-822, 2008.

[28] P. Nangia-Makker, L. Tait, M. P. V. Shekhar et al., "Inhibition of breast tumor growth and angiogenesis by a medicinal herb: Ocimum gratissimum," International Journal of Cancer, vol. 121, no. 4, pp. 884-894, 2007. 


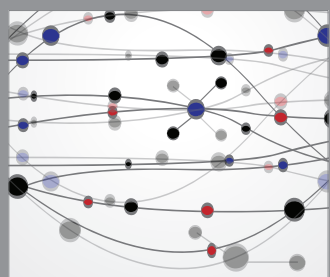

The Scientific World Journal
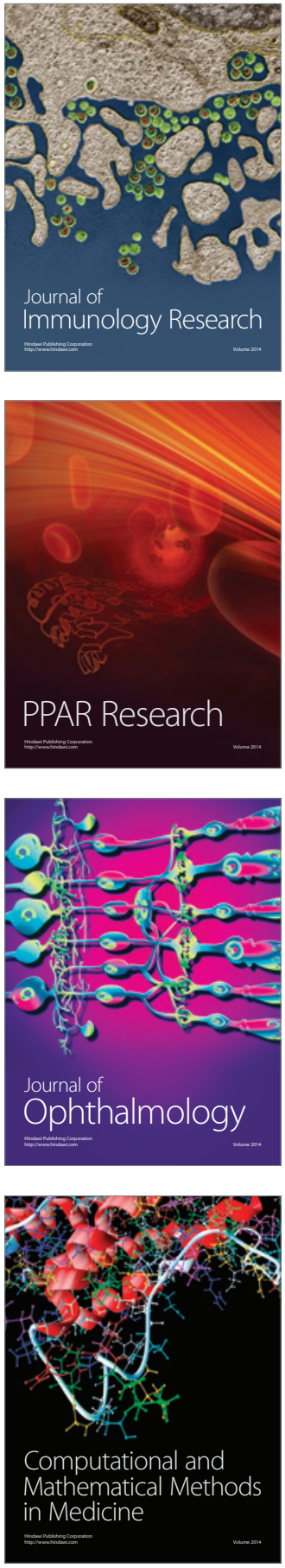

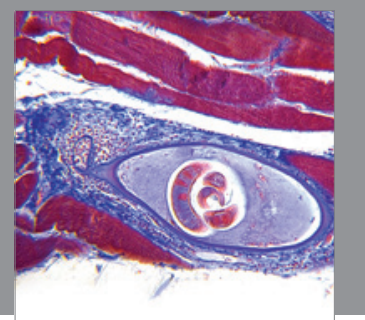

Gastroenterology

Research and Practice
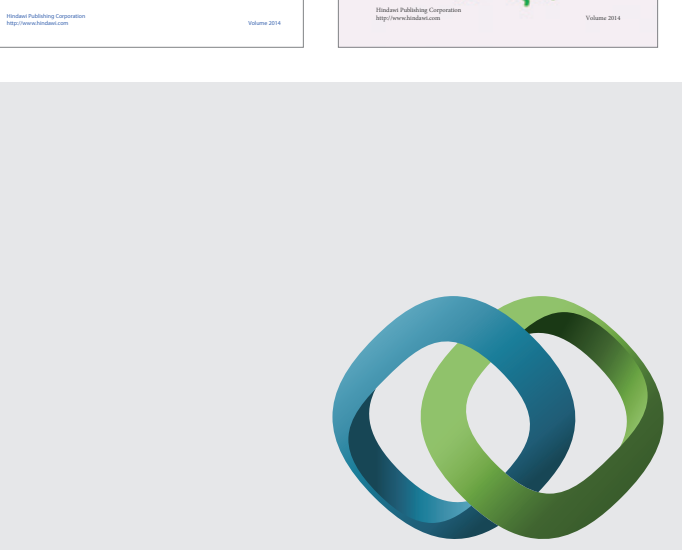

\section{Hindawi}

Submit your manuscripts at

http://www.hindawi.com
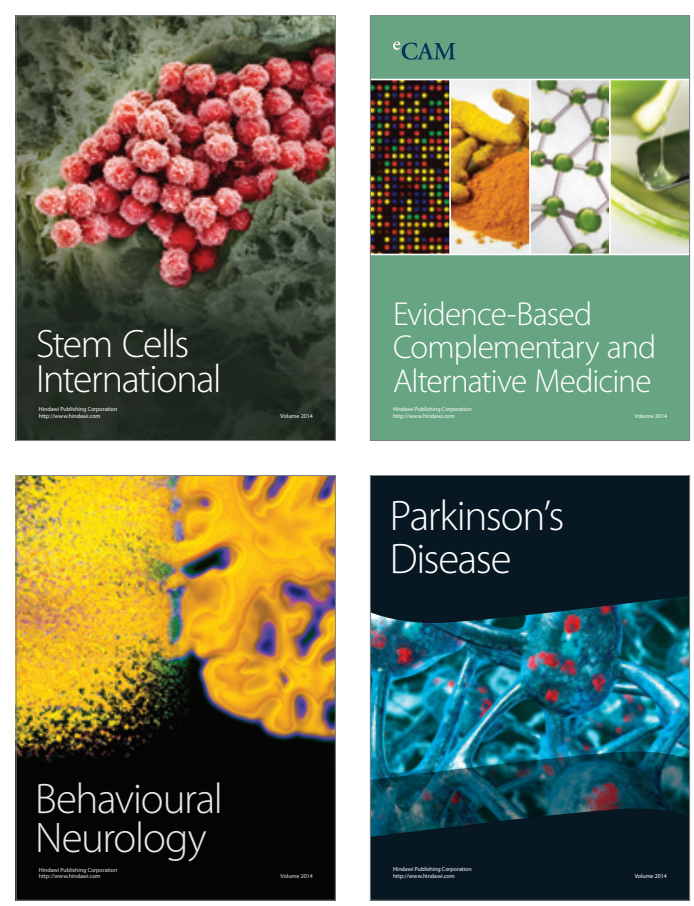

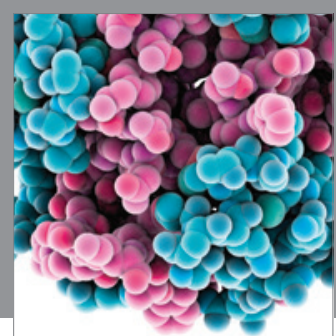

Journal of
Diabetes Research

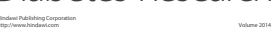

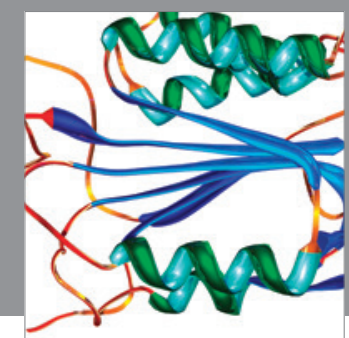

Disease Markers
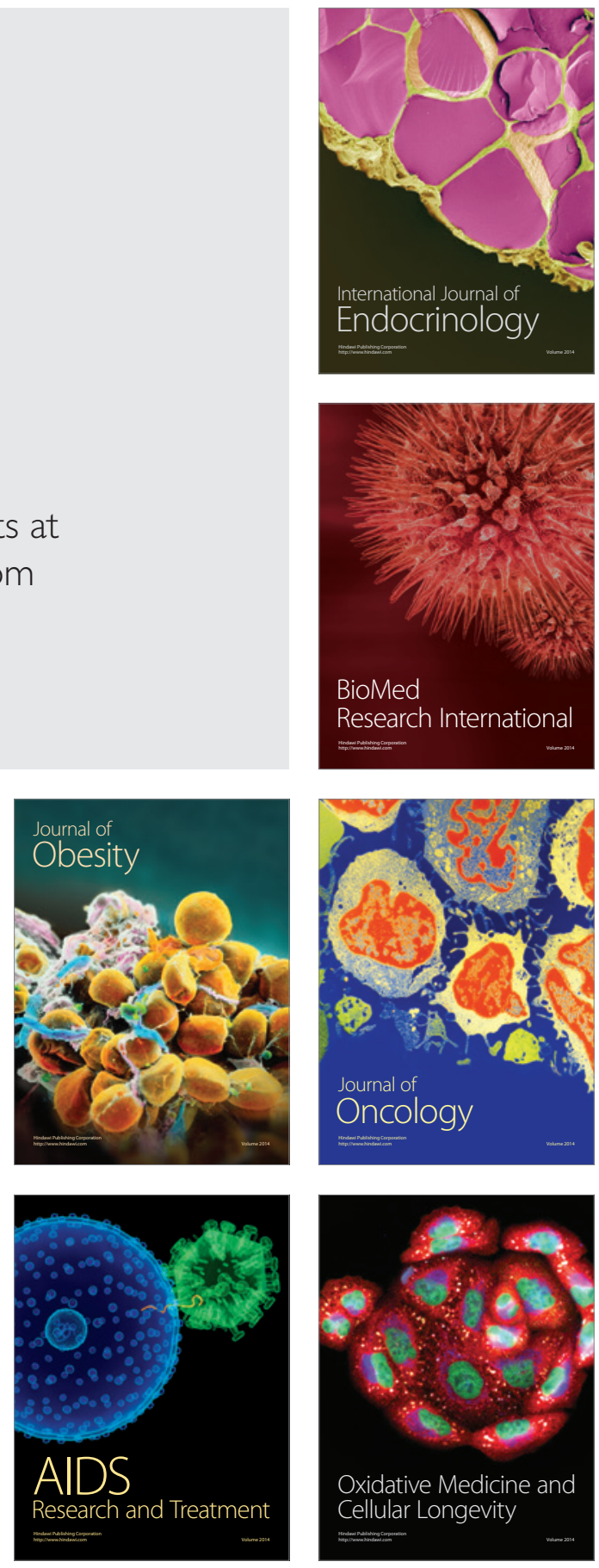